\title{
Effet de la forme de présentation de l'orge sur la production et la composition du lait de vache*
}

\author{
JB Coulon 1, C Agabriel 2 \\ avec la collaboration technique de JC Bonnefoy \\ 1 INRA-Theix, laboratoire d'adaptation des herbivores aux milieux, \\ 63122 Saint-Genès-Champanelle; \\ 2 ENITA-Clermont-Ferrand, 63370 Lempdes, France
}

(Reçu le 27 septembre 1994; accepté le 17 octobre 1994)

\begin{abstract}
Résumé - L'effet de la forme de présentation de l'orge (broyée (B) ou aplatie (A)) a été étudiè sur 22 vaches multipares en phase descendante de lactation, utilisées dans un essai en inversion avec 2 périodes successives de $3 \mathrm{sem}$. Au cours de l'essai, tous les animaux ont reçu une ration constituée d'un mélange 75/15/10 d'ensilage de maïs, d'ensilage d'herbe et de drèches de brasserie, et de foin offert à volonté, ainsi que de $2 \mathrm{~kg}$ de pulpes déshydratées. Les quantités d'aliment concentré fermier (mélange 75/25 d'orge et de tourteaux) ont été calculées en fonction du niveau de production des animaux et n'ont pas varié au cours de l'essai. Dans le traitement $\mathrm{B}, 100 \mathrm{~g}$ de substances tampons ont été ajoutés à la ration. En moyenne, les animaux ont ingéré $8,2 \mathrm{~kg} / \mathrm{j}$ de concentrés, dont $4,7 \mathrm{~kg}$ d'orge. La forme de présentation de l'orge n'a eu d'effet ni sur la vitesse d'ingestion du concentré $(240 \mathrm{~g} / \mathrm{min}$ en moyenne), ni sur la production laitière (respectivement 33,1 et $32,7 \mathrm{~kg} / \mathrm{j}$ dans les lots $B$ et $A$ ), ni sur la composition chimique du lait, et en particulier le taux butyreux $(38,4$ et 37,7 dans les lots $B$ et A). Le $\mathrm{pH}$ du jus de rumen et la proportion des principaux acides gras volatils du rumen ont été semblables dans les 2 lots.
\end{abstract}

\section{vache laitière / concentré / forme de présentation}

Summary - Effect of grain processing on milk production and composition. The effect of grain processing (ground [G] or rolled [R]) was analysed on 22 multiparous cows in mid-lactation used in a $2 * 2$ latin-square design experiment. During each 3-wk period, cows were offered a diet composed of a mixture of corn silage, grass silage and brewer's grain (75/15/10, ad libitum), of hay (ad libitum) and of dried sugar beet pulp (2 kg/d). The quantities of concentrate ( $75 \%$ barley and $25 \%$ meal) were calculated according to milk production level and did not vary during the trial. In group G, $100 \mathrm{~g} / \mathrm{d}$ of buffer compound was added to the diet. Grain processing had effect neither on time spent to eat the

\footnotetext{
* Cette étude a été réalisée avec la participation financière de l'association Gala.
} 
concentrate (4.1 min/kg on average) nor on milk yield (33.1 and $32.7 \mathrm{~kg} / \mathrm{d}$, respectively, in group $\mathrm{G}$ and R) nor on milk fat content ( 38.4 and $37.7 \mathrm{~g} / \mathrm{kg}$, respectively). $\mathrm{pH}$ of rumen juice and concentrations of volatile fatty acids were identical in the 2 groups.

dairy cow / concentrate / grain processing

\section{INTRODUCTION}

Le taux butyreux du lait devient l'objet d'enjeux économiques importants. Ses principaux facteurs de variation d'origine alimentaire (proportion de concentré dans la ration, nature et modalités de distribution des aliments) sont bien connus (Journet et Chilliard, 1985 ; Sutton, 1989 ; Hoden et Coulon, 1991). Ces facteurs permettent généralement d'expliquer correctement les variations, parfois considérables, du taux butyreux d'une exploitation à l'autre (Agabriel et al, 1993a). Cependant, dans certaines situations, ces variations restent difficiles à expliquer. Ainsi, des écarts importants de taux butyreux (environ $3 \mathrm{~g} / \mathrm{kg}$ à l'échelle de l'année) ont été observés, à même niveau génétique, dans des exploitations où les principales caractéristiques alimentaires (type de ration de base, quantité et nature de l'aliment concentré) semblaient relativement homogènes (Agabriel et al, 1993b). Ces variations ont pu être associées à l'ordre de distribution des aliments dans la journée et à la forme de présentation des céréales (aplatie ou broyée) (Coulon et al, 1994), qui sont connus pour pouvoir modifier les orientations fermentaires dans le rumen (Orskov, 1979 ; Klusmeyer et al, 1990 ; Nocek, 1992). Ceci doit être d'autant plus vraisemblable que les animaux sont plus forts producteurs et reçoivent donc des quantités plus importantes de concentrés. II existe cependant peu de travaux expérimentaux récents sur ces thèmes chez la vache laitière (cf revues de Campling, 1991, et Nocek, 1992). L'objectif de cette étude est d'analyser expérimentalement l'effet de la forme de présentation des céréales sur le taux butyreux du lait chez des animaux à haut niveau de production.

\section{MATÉRIEL ET MÉTHODES}

\section{Schéma expérimental et alimentation}

Cette étude a été réalisée sur les vaches d'une exploitation privée du Puy-de-Dôme, située à une altitude de $650 \mathrm{~m}$. Le troupeau est constitué de 53 vaches Montbéliardes, ayant produit, en moyenne en $1993,8160 \mathrm{~kg}$ de lait à $39,9 \mathrm{~g} / \mathrm{kg}$ de taux butyreux et $33,5 \mathrm{~g} / \mathrm{kg}$ de taux protéique (résultats Contrôle laitier 1993). Aucune modification de la conduite des animaux, et en particulier de leur alimentation, n'a été apportée en dehors de la forme de présentation des céréales de la ration.

Vingt-deux vaches multipares ayant vêlé entre le 31 juillet et le 31 décembre (27 octobre en moyenne) ont été utilisées. Au cours de l'hiver, les animaux, conduits en stabulation entravée et traits en place, ont reçu une ration constituée d'un mélange 75/15/10 d'ensilage de maïs, d'ensilage d'herbe et de drêches de brasseries, distribué le matin après la traite, et de foin de prairie permanente de deuxième coupe (offert à volonté, quantité ingérée estimée à $6 \mathrm{~kg} / \mathrm{vache} / \mathrm{j}$ ), distribué le soir après la traite. Par ailleurs, $2 \mathrm{~kg}$ de pulpes de betteraves désydratées ont été distribués $(1 \mathrm{~kg}$ le matin et $1 \mathrm{~kg}$ le soir). L'aliment concentré (mélange $75 / 25$ d'orge broyée et d'un mélange de tourteau tanné - $60 \%$ soja, $20 \%$ colza et $20 \%$ lin) était offert en 2 repas, avant les traites, à raison de $1 \mathrm{~kg}$ par tranche de $2 \mathrm{~kg}$ de lait au dessus de $20 \mathrm{~kg} / \mathrm{j}$. Les animaux recevaient par ailleurs $80 \mathrm{~g} / \mathrm{j}$ d'un complément minéral (15P-21Ca), $25 \mathrm{~g} / \mathrm{j}$ d'un additif à base de probiotiques et $100 \mathrm{~g} / \mathrm{j}$ d'un mélange 50/25/25 de bicarbonate de sodium, magnésie et carbonate de calcium. L'essai, organisé selon un schéma en inversion avec 2 périodes successives de 3 sem, a débuté le 17 janvier. Deux lots de 11 vaches ont été constitués sur la base de la date de vêlage des ani- 
maux, de la production et de la composition du lait la semaine précédant le début de l'essai, du poids vif et du numéro de lactation. Au cours des $6 \mathrm{sem}$ expérimentales les mêmes silos et le même lot de foin ont été utilisés, et la quantité de concentré offerte à chaque animal expérimental a été maintenue constante.

Jusqu'au 16 janvier, tous les animaux ont reçu les céréales sous forme broyée. Entre le 17 janvier et le 6 février, le premier lot a continué de recevoir les céréales sous forme broyée (lot $\mathrm{B}$ ), tandis que l'autre les recevait sous forme aplatie (lot A). Entre le 7 et le 27 février les traitements ont été inversés. À partir du 28 février, tous les animaux ont reçu à nouveau les céréales sous forme broyée. Lorsque les céréales étaient distribuées sous forme aplatie, les $100 \mathrm{~g}$ du mélange de substances tampons défini précédemment n'ont pas été distribués aux animaux. Seuls $100 \mathrm{~g} / \mathrm{sem}$ de magnésie ont été offerts. Le passage d'un mode de présentation à l'autre s'est fait du jour au lendemain. Les céréales ont été aplaties à l'aide d'un aplatisseur à 2 rouleaux.

\section{Mesures}

La production laitière et la composition chimique du lait ont été mesurées individuellement 1 fois par semaine sur 2 traites consécutives pendant les 2 premières sem de chaque période et 2 fois par semaine la dernière semaine de chaque période. Les animaux ont été pesés et leur état corporel a été noté (par maniement) 2 fois au cours de l'essai, entre le 7 et le 15 janvier et entre le 28 février et le 6 mars. Les quantités de concentrés offertes ont été mesurées individuellement tous les jours. Un prélèvement rectal de fécès a été réalisé sur tous les animaux au cours de la dernière semaine de chaque période. Le $\mathrm{pH}$ a été immédiatement mesuré, et un échantillon a été constitué pour analyse des teneurs en matière sèche, en amidon et en matières azotées totales. Une fois au cours de la dernière semaine de chacune des 2 périodes expérimentales, un prélèvement de jus de rumen a été réalisé sur 7 vaches de chaque lot, par ponction abdominale. Le $\mathrm{pH}$ a été immédiatement mesuré, et un échantillon additionné de conservateur a été constitué et congelé pour analyse de la composition du mélange des AGV. Une fois au cours de la dernière semaine de chaque période, le comportement individuel des 22 vaches a été observé, par pointage, toutes les 5 min pendant les 3 h suivant la distribution des aliments du matin et du soir, des principales activités des animaux (debout, couché, ingestion de fourrage, ingestion de concentré, consommation d'eau, consommation de paille, rumination). Le temps mis pour ingérer la totalité du concentré a été noté.

Sur un échantillon de chacun des fourrages utilisés et sur un échantillon de chacun des concentrés utilisés, les teneurs en matière sèche, en matière organique, en cellulose brute et en matières azotées totales ont été déterminées. Sur l'ensilage de maïs on a déterminé en plus la teneur en amidon. Les caractéristiques des aliments sont précisées au tableau I. Une mesure de la granulométrie a été réalisée au cours de chaque période sur chacune des 2 formes de présentation des céréales ; $66 \%$ des particules de céréales aplaties avaient une taille supérieure à $1 \mathrm{~mm}$ et $25 \%$ une taille supérieure à $4 \mathrm{~mm}$. Aucune particule des céréales broyées ne présentait une taille supérieure à $4 \mathrm{~mm}, 32 \%$ avaient une taille comprise entre 1 et $4 \mathrm{~mm}$ et $19 \%$ avaient une taille inférieure à $0,4 \mathrm{~mm}$.

L'effet de la forme de présentation sur les différentes variables étudiées a été traité par analyse de variance sur les mesures effectuées au cours de la dernière semaine de chaque période expérimentale. Les facteurs introduits dans l'analyse ont été le traitement, la période et l'animal.

\section{RÉSULTATS}

En début d'expérience, les animaux pesaient en moyenne $690 \mathrm{~kg}$ et étaient en bon état corporel (note de 2,7 en moyenne). Ils ont gagné un peu de poids et d'état au cours de l'essai (respectivement $+11 \mathrm{~kg}$ et $+0,2$ point d'état). Au cours de la période expérimentale, les animaux ont reçu en moyenne $8,2 \mathrm{~kg} / \mathrm{j}$ d'aliments concentrés (pulpes + mélange d'orge et de tourteaux) dont $4,7 \mathrm{~kg} / \mathrm{j}$ d'orge. Chez les 6 animaux les plus forts producteurs (>38 kg/j), ces quantités ont atteint respectivement $11,3 \mathrm{et} 7 \mathrm{~kg} / \mathrm{j}$. Bien que les quantités de fourrages ingérées n'aient pas été mesurées, on peut estimer (Faverdin et al, 1987 ; Coulon et Faverdin, 1994), compte tenu des caractéristiques des aliments, que l'ensemble des concen- 
Tableau I. Caractéristiques des aliments.

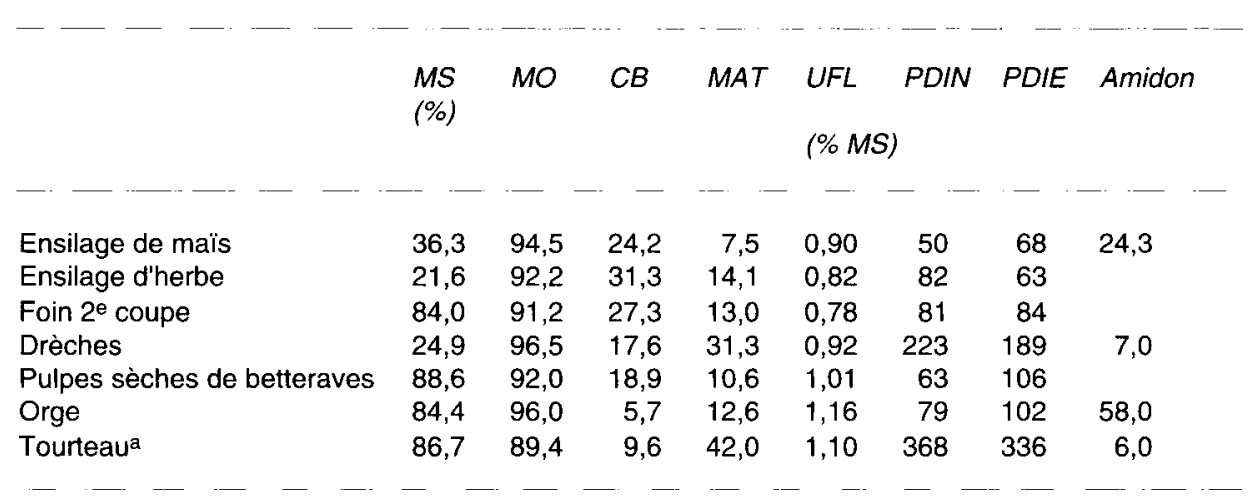

a $60 \%$ soja, $20 \%$ colza, $20 \%$ lin.

trés ont représenté entre 20 et $45 \%$ de la MS de la ration totale selon le niveau de production des animaux ( $32 \%$ en moyenne).

Le temps d'ingestion du concentré a varié selon les animaux de 10 à $27 \mathrm{~min}$. Cette variabilité a été indépendante des quantités de concentrés offertes et de la forme de présentation des céréales (respectivement 16,9 et 16,6 min en moyenne dans les lots B et A pour $4,1 \mathrm{~kg}$ de concentré par repas en moyenne, soit 4,1 $\mathrm{min} / \mathrm{kg}$ dans les 2 lots). Le temps passé à ingérer du fourrage au cours des 60 minutes suivant leur distribution a été légèrement supérieur dans le lot $B$ (38 contre 33 min dans le lot $A, P<0,01$ ).

La forme de présentation des céréales n'a eu d'effet significatif ni sur la production laitière (respectivement 33,1 et $32,7 \mathrm{~kg} / \mathrm{j}$ dans les lots $B$ et $A$ ), ni sur la composition chimique du lait (respectivement 38,4 et $37,7 \mathrm{~g} / \mathrm{kg}$ pour le taux butyreux dans les lots $B$ et $A$ et 32,1 et $32,4 \mathrm{~kg}$ pour le taux protéique (tableau II). En conséquence, les quantités de matières produites ont été semblables dans les 2 lots (respectivement 1267 et $1225 \mathrm{~g} / \mathrm{j}$ pour les matières grasses dans les lots $B$ et $A$, et 1059 et $1052 \mathrm{~g} / \mathrm{j}$ pour les matières protéiques). Les variations inter-individuelles du taux butyreux n'ont pas été liées à celles des quantités de concentrés offertes.

Le $\mathrm{pH}$ du jus de rumen n'a pas été significativement différent d'un traitement à l'autre (respectivement 5,79 et 5,69 ). Ces faibles valeurs du $\mathrm{pH}$ du jus de rumen sont liées en grande partie à l'heure de prélèvement ( 3 à $4 \mathrm{~h}$ après la distribution des aliments, c'est-à-dire au moment où le pH est le plus faible (Sutton, 1981)) et à l'endroit du prélèvement, dans le sac dorsal, où le $\mathrm{pH}$ est le plus faible (inférieur de 0,3 à 0,4 unité à celui observé dans le sac ventral (Lampila et Poutianen, 1966 ; Brugère et al, 1990)). La proportion relative des principaux acides gras volatils du jus de rumen a été identique dans les 2 traitements (tableau II) et peu variable d'une vache à l'autre : 26 des 30 prélèvements réalisées ont présenté un rapport (acide acétique + acide butyrique)/acide propionique compris entre 3,5 et 4,2. Dans ces conditions, les variations individuelles du taux butyreux n'ont pas été liées à ce rapport.

Le $\mathrm{pH}$ des bouses, leur teneur en MS et en matières azotées totales ont été voisins dans les 2 traitements, de même que la teneur en amidon, faible en moyenne (1,8\% MS). Ces caractéristiques n'ont pas varié 
Tableau II. Performances des animaux, caractéristiques des bouses et du jus de rumen selon les lots.

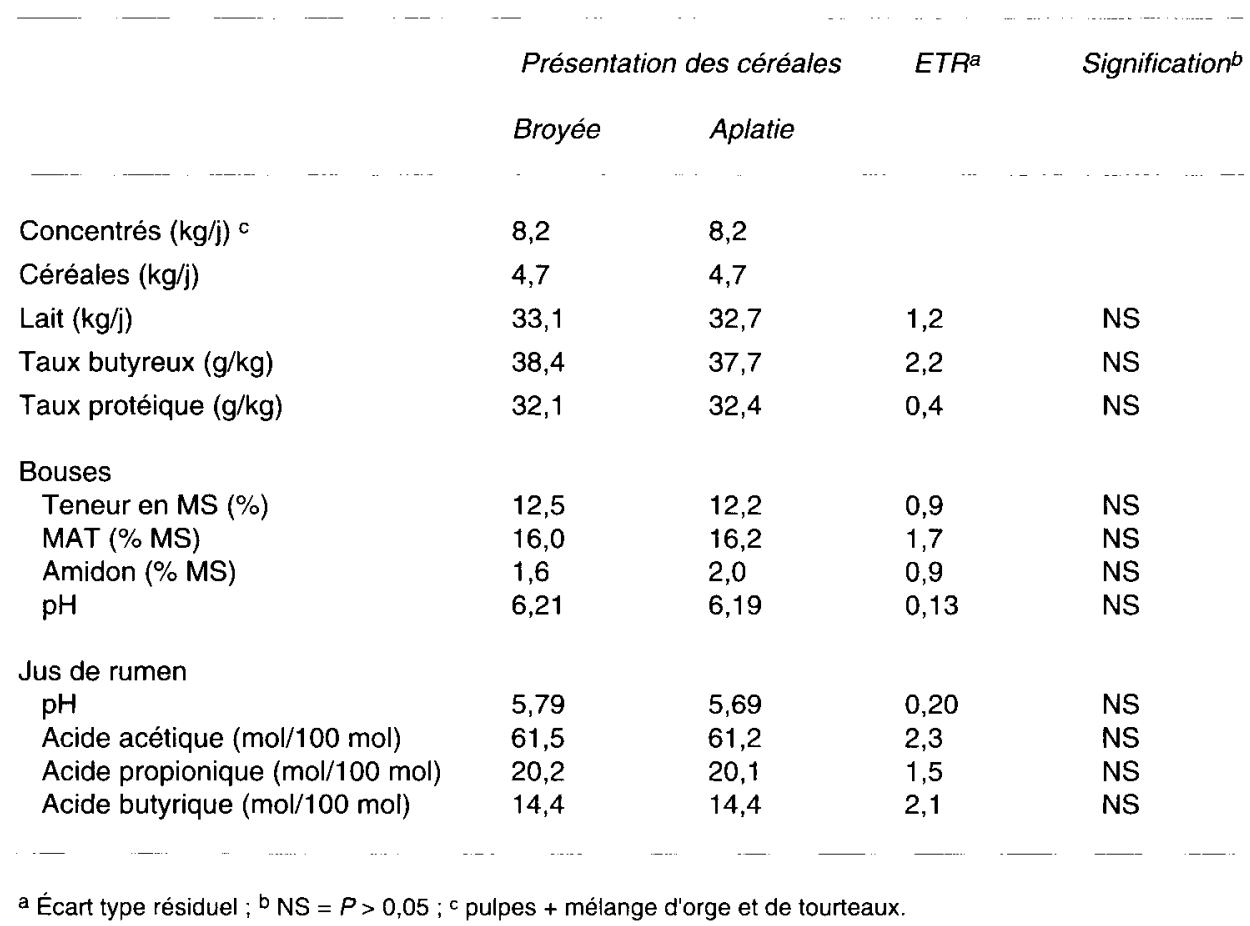

en fonction de la quantité de concentré offerte.

Cette absence d'écart significatif sur les performances de production et sur les caractéristiques du jus de rumen et des bouses persiste si l'on ne considère plus que les 13 vaches ayant reçu plus de $7 \mathrm{~kg}$ de concentré par jour $(9,8 \mathrm{~kg} / \mathrm{j}$ en moyenne dont 5,9 de céréales).

Le changement de forme de présentation des céréales s'est accompagné chez la plupart des animaux d'une diminution importante mais fugace de la production laitière au cours des 2 j suivant le changement. Cette diminution a été plus importante lorsque les animaux sont passés de la forme broyée à la forme aplatie $(3,7 \mathrm{~kg}$ de chute de lait en moyenne entre le dernier jour de distribution de la forme broyée et le $2^{\mathrm{e}}$ jour de distribution de la forme aplatie) que lorsqu'ils sont passés de la forme aplatie à la forme broyée $(1,5 \mathrm{~kg}$ de chute de lait). Ces modifications de la production n'ont pas été accompagnées de refus de concentrés les jours suivant le changement de forme de présentation. Elles sont vraisemblablement dues à une perturbation temporaire de la digestion dans le rumen. Cette réaction importante des animaux au changement d'alimentation montre la sensibilité d'animaux forts producteurs à des modifications apparemment limitées de l'alimentation. Elle a certainement été accentuée par le fait qu'ils n'avaient pas été habitués auparavant à recevoir des céréales sous forme aplatie. 


\section{DISCUSSION ET CONCLUSION}

Les résultats de cette étude sont en accord avec les travaux réalisés sur des rations à base d'ensilage de maïs en milieu de lactation qui n'avaient pas mis en évidence d'effet significatif de la forme de présentation des céréales (blé aplati ou broyé) sur les performances des animaux, contrairement à ce qui était observé en début de lactation (Laurent, 1988 ; Cotto, 1991). L'absence de différence observée entre les 2 traitements peut s'expliquer par différentes raisons. En premier lieu, la ration utilisée était constituée d'une quantité importante de fibres, en particulier sous forme de foin offert à volonté. En moyenne, sa teneur en cellulose brute peut être estimée à $20 \%$, sa teneur en amidon à $20 \%$ et son indice de fibrosité, calculée à partir des données de Sauvant et al (1990), à $50 \mathrm{~min} / \mathrm{kg} \mathrm{MS}$. Même chez les animaux les plus fort producteurs, où ces valeurs atteignent respectivement $18 \%, 23 \%$ et $45 \mathrm{~min} / \mathrm{kg} \mathrm{MS}$, ces valeurs restent élevées, supérieures à celles à partir desquelles les risques de chute de taux butyreux sont observés (Journet et Chilliard, 1985 ; Sutton et Morant, 1989 ; Sauvant et al, 1990). Les liaisons entre les variations du taux butyreux du lait et la teneur en cellulose brute de la ration sont cependant très variables (Journet et Chilliard, 1985). En second lieu, le broyage de l'orge utilisée, bien qu'assez important, a été un peu moins fin que celui généralement pratiqué par les industriels lorsqu'ils agglomèrent les céréales, ce qui a pu limiter l'éventuel effet dépressif du broyage sur le taux butyreux. La finesse de broyage de la ration est en effet un facteur important de variation du taux butyreux (Grant et al, 1990 ; Klusmeyer et al, 1990). Enfin, la présence de substances tampons dans le lot broyé a pu permettre de limiter les risques de diminution excessive du $\mathrm{pH}$ du rumen et de chute du taux butyreux. L'effet favorable des substances tampons sur le taux buty- reux est en effet bien connu, lorsque ces taux sont faibles. Cependant, lorsqu'ils atteignent un niveau de l'ordre de $35 \mathrm{~g} / \mathrm{kg}$, cet effet disparaît généralement (Journet et Chilliard, 1985 ; Xu et al, 1994).

Ces résultats ne permettent pas de confirmer les observations faites dans le cadre de l'enquête effectuée dans des exploitations utilisant des rations à base de foin et qui avaient suggéré un effet favorable de la forme aplatie sur le taux butyreux du lait (Coulon et al, 1994). II est cependant possible que cet effet soit plus sensible sur des rations à base de foin que sur des rations à base d'ensilage de maïs. Celles-ci sont en effet connues pour favoriser le taux butyreux du lait (Hoden et al, 1985), en raison des orientations fermentaires dans le rumen et de la richesse en lipides du grain de mais. D'autre part, dans les exploitations enquêtées, d'autres facteurs identifiés (séquence de distribution des différents types d'aliments) ou non ont pu interagir avec la forme de présentation des céréales. Nous ne sommes cependant pas parvenu à mettre en évidence, en station expérimentale, l'effet de l'ordre de distribution des aliments sur le taux butyreux du lait (Chassaing et al, 1995).

En définitive, cet essai permet de montrer que, chez des animaux à haut niveau de production ingérant jusqu'à $7 \mathrm{~kg}$ de céréales par jour, et dans des conditions de conduite optimales (l'effet troupeau de l'exploitation était égal, en 1992, à respectivement $2816 \mathrm{~kg}, 1,9 \mathrm{~g} / \mathrm{kg}$ et $1,5 \mathrm{~g} / \mathrm{kg}$ pour la production laitière, le taux butyreux et le taux protéique), l'utilisation des céréales sous forme aplatie permet, une fois les animaux habitués à ce nouvel aliment, des performances zootechniques équivalentes à celles obtenues avec la forme broyée, en évitant de plus l'adjonction de substances tampons à la ration. Dans ces conditions, le choix d'une forme ou l'autre doit prendre en compte non seulement un facteur de risque de dysfonctionnement du rumen (a priori 
plus important avec la forme broyée) que ce type d'expérimentation ne permet pas de mettre en évidence, mais aussi des facteurs économiques ou liés à l'organisation du travail (coût respectif des 2 formes de présentation, travail nécessaire à leur réalisation sur l'exploitation, facilité de stockage et de distribution...).

\section{REMERCIEMENTS}

Nous tenons à remercier $P$ et $M$ Giraud qui ont permis la réalisation de cette expérimentation dans leur exploitation et en ont accepté les contraintes, ainsi que $C$ Cougoul qui a réalisé les prélèvements de lait, $M$ Ledeux et $A$ Debiasi qui ont réalisé les prélèvements de jus de rumen, $J$ Chabrot pour son aide dans la mise en œuvre des analyses chromatographiques, et $A$ Jordan pour sa participation aux observations de comportement alimentaire. Nous remercions aussi la société Sormag qui a mis à notre disposition l'aplatisseur pour la durée de l'expérimentation.

\section{RÉFÉRENCES}

Agabriel C, Coulon JB, Marty G, Bonaïti B, Boniface P (1993a) Effets respectits de la génétique et du milieu sur la production et la composition du lait de vache. Étude en exploitations. INRA Prod Anim 6, 213-223

Agabriel C, Coulon JB, Marty G, Bonaïti B (1993b) Changes in fat and protein concentrations in farms with high milk production. J Dairy Sci 76, 734-741

Brugère H, Champy R, Polack B, Rousseau JF (1990) Examen du jus de rumen et surveillance de l'alimentation des Bovins. Apports de la titrimétrie. Rec Méd Vét 166, 133-142

Campling RC (1991) Processing cereal grains for cattle. A review. Livest Prod Sci 28, 223-234

Chassaing C, Coulon JB, Agabriel C, Garel JP (1995) The effect of feeding sequence on fat concentration in milk. Ann Zootech (à paraître)

Cotto $G$ (1991) L'utilisation des céréales par les vaches laitières. ITEB, Paris, $48 p$

Coulon JB, Agabriel C, Brunschwig G, Muller C, Bonaiti B (1994) Effects of feeding practices on milk fat concentration for dairy cows. J Dairy Sci 77, 26142620
Coulon JB, Faverdin P (1994) Vaches laitières. In : INRAtion, logiciel de calcul et d'analyse de rations pour les ruminants, version 2.5 (J Agabriel, P Champciaux, C Espinasse, eds), CNERTA, Dijon

Faverdin P, Hoden A, Coulon JB (1987) Recommandations alimentaires pour les vaches laitières. Bull Tech CRZV Theix INRA 70, 133-152

Grant RJ, Colenbrander VF, Albright JL (1990) Effect of particule size of forage and rumen cannulation upon chewing activity and laterality in dairy cows. $J$ Dairy Sci 73, 3158-3164

Hoden A, Coulon JB, Dulphy JP (1985) Influence de l'alimentation sur la composition du lait. III. Effets des régimes alimentaires sur les taux butyreux et protéiques. Bull Tech CRZV Theix INRA 62, 69-79

Hoden A, Coulon JB (1991) Maîtrise de la composition du lait : influence des facteurs nutritionnels sur la quantité et les taux de matières grasses et protéiques. INRA Prod Anim 4, 361-367

Journet M, Chilliard $Y$ (1985) Influence de l'alimentation sur la composition du lait. 1. Taux butyreux : facteurs généraux. Bull Tech CRZV Theix INRA 60, 1323

Klusmeyer TH, Cameron MR, McCoy GC, Clark JH (1990) Effects of feed processing and frequency of feeding on ruminal fermentation, milk production and milk composition. J Dairy Sci 73, 3538-3543

Lampila M, Poutianen E (1966) Systematic differences in the composition of the bovine rumen fluid between different parts of the rumen. Ann Agric Fenn 5 , 279-297

Laurent $F$ (1988) Utilisation du blé et des céréales dans la ration des vaches laitières. Ann Zootech 37, 117132

Nocek JE (1992) Feeding sequence and strategy effects on ruminal environment and production performance in first lactation cows. J Dairy Sci 75, 3100-3108

Orskov ER (1979) Recent information on processing of grain for ruminants. Livest Prod Sci 6, 335-347

Sauvant D, Dulphy JP, Michalet-Doreau B (1990) Le concept d'indice de fibrosité des aliments des ruminants. INRA Prod Anim 3, 309-318

Sutton JD (1981) Concentrate feeding and milk composition. In : Recent advances in animal nutrition ( $W$ Haresign, ed), Butterworths, London, 35-48

Sutton JD (1989) Altering milk composition by feeding J Dairy Sci 72, 2801-2814

Sutton JD, Morant SV (1989) A review of the potential of nutrition to modify milk fat and protein. Livest Prod SCi 23, 219-237

Xu S, Harrisson JH, Riley RE, Loney KA (1994) Effect of buffer addition to high grain total mixed rations on rumen $\mathrm{pH}$, feed intake, milk production, and milk composition. J Dairy Sci 77, 782-788 